\title{
Indicadores antropométricos na avaliação nutricional de idosos: um estudo comparativo
}

\author{
Anthropometric indicators in the nutritional \\ assessment of the elderly: a comparative study
}

Analia Nusya de Medeiros GARCIA

Sylvia de Azevedo Mello ROMANI ${ }^{2}$

Pedro Israel Cabral de LIRA2

\section{R E S U M O}

\section{Objetivo}

Comparar as medidas antropométricas da envergadura e circunferência braquial para avaliação do estado nutricional de idosos em relação ao Índice de Massa Corporal.

\section{Métodos}

Foram estudados 308 idosos residentes em instituições asilares públicas e particulares em Pernambuco, Brasil.

\section{Resultados}

A sensibilidade do Índice de Massa Corporal, tendo a envergadura como preditora da estatura, no diagnóstico da desnutrição, foi de 93,5\%, com especificidade de 82,0\%. Com relação ao diagnóstico de sobrepeso e obesidade, o cálculo do Índice de Massa Corporal, utilizando a envergadura, demonstrou sensibilidade de $44,5 \%$ e $83,3 \%$ e especificidade de $87,0 \%$ e $100,0 \%$, respectivamente. A circunferência braquial apresentou, no diagnóstico de desnutrição, sensibilidade e especificidade de $89,0 \%$ e $87,0 \%$, respectivamente.

\section{Conclusão}

Em idosos, o uso da envergadura em substituição à altura no cálculo do Índice de Massa Corporal, mostrou-se um bom indicador no diagnóstico de desnutrição e de obesidade, no entanto não se observou o mesmo em relação ao diagnóstico de sobrepeso. A medida da circunferência braquial foi também um bom indicador para determinar desnutrição em idosos.

Termos de indexação: circunferência braquial; idoso; índice de massa corporal; antropometria.

\section{A B S T R A C T}

\section{Objective}

This study compared anthropometric measurements (arm span and circumference) used in the assessment of nutritional status of the elderly in relation to Body Mass Index.

\footnotetext{
1 Universidade de Pernambuco, Instituto de Ciências Biológicas. R. Megina Pontual, 147, Bloco 1001, Boa Viagem, 51021-510, Recife PE, Brasil. Correspondência para/ Correspondence to: A.N.M. GARCIA. E-mail:<analiag@oi.com.br>.

2 Universidade Federal de Pernambuco, Departamento de Nutrição. Recife, PE, Brasil.
} 


\section{Methods}

The sample consisted of 308 elderly living in private and public nursing homes in the State of Pernambuco, northeast Brazil.

\section{Results}

Body Mass Index sensitivity with height being determined by arm span in the diagnosis of malnutrition was $93.5 \%$, with a specificity of $82 \%$. With regard to the diagnosis of overweight and obesity, the sensitivity was $44.5 \%$ and $83.3 \%$ and specificity $87.0 \%$ and $100.0 \%$ respectively. Among those diagnosed with malnutrition, arm circumference showed a sensitivity and specificity of $89.0 \%$ and $87.0 \%$ respectively.

\section{Conclusion}

We conclude that using arm span as a proxy for height when calculating Body Mass Index is a good indicator of malnutrition and obesity; however, the same was not observed for the overweight. Arm circumference was also found to be a good indicator of malnutrition in the elderly.

Indexing terms: mid-upper arm circunference; elderly; body mass index; anthropometry.

\section{N T R O D U Ç Ã O}

O envelhecimento populacional é hoje um fenômeno universal, característico tanto dos países desenvolvidos como, de modo crescente, dos países em desenvolvimento'. A população mundial total deverá crescer $80 \%$ (passando de 4,4 para 7,8 bilhões), enquanto o número de idosos deverá crescer $160 \%^{2}$. Na América Latina e Caribe, durante o período de 1980 a 2025, a população de 60 anos e mais terá, em média, dobrado, pelo menos, uma vez ${ }^{3}$.

O envelhecimento afeta diretamente o estado nutricional do indivíduo por todas as alterações que ocorrem no organismo, tais como, diminuição dos botões gustativos, redução do olfato e da visão, diminuição da secreção salivar e gástrica, falha na mastigação (pela ausência de dentes ou próteses impróprias), constipação intestinal devido à redução da motilidade. Estudos epidemiológicos, também, indicam que, especialmente, em homens idosos a desnutrição reduz significativamente o tempo de vida ${ }^{4-6}$.

A pessoa idosa apresenta tendência a desenvolver desnutrição, devido à incidência de doenças crônicas e debilidades físicas associadas à idade 7 . Alterações nutricionais podem contribuir ou ainda, exacerbar doenças crônicas e agudas, sejam de origem física ou mental, acelerar o desenvolvimento de doenças degenerativas, dificultando o processo de recuperação das mesmas. O distúrbio nutricional mais importante observado nos idosos é a desnutrição proteico-calórica (DPC), que está associada ao aumento da mortalidade e da susceptibilidade às infecções e à redução da qualidade de vida ${ }^{8}$.

A avaliação do estado nutricional é importante para o diagnóstico da desnutrição, principalmente nas fases iniciais ou ainda quando há dúvidas na interpretação de dados subjetivos. Para avaliação do estado nutricional de idosos, utilizam-se parâmetros, como história clínica, dietética, exames laboratoriais e medidas antropométricas, para que se possa, efetivamente, chegar a um diagnóstico com segurança. Pesquisadores têm envidado esforços no sentido de desenvolver um modelo de avaliação utilizável em qualquer parte do mundo, de metodologia simples, facilmente reprodutível e que possa ser executado a baixo custo na maioria das localidades ${ }^{7,9}$.

Nesse sentido, existe a antropometria como um método não invasivo, de fácil execução, de baixo custo operacional, seguro e que tem valor preditivo acurado para identificar populações em risco nutricional ${ }^{10-12}$.

$\mathrm{Na}$ avaliação antropométrica da população idosa incluem-se, além das medidas da altura e do peso, objetivando o cálculo do Índice de Massa Corporal (IMC), a circunferência do braço. Caso o indivíduo não tenha condições de se levantar, ficar em pé, possua deformidade na coluna vertebral ou amputação de uma perna, os autores indicam fazer uma estimativa da altura 
por meio de equações específicas, utilizando-se o comprimento da ulna, perônio ou envergadura dos braços $^{7,13}$.

A medida da envergadura é uma alternativa a mais, no estudo antropométrico, principalmente, quando da necessidade de avaliar indivíduos em cadeiras de rodas ou acamados, embora apresente algumas dificuldades na execução da medida, podendo diminuir a acurácia do método ${ }^{14}$.

A carência de estudos metodológicos e epidemiológicos nessa área, no Brasil, e especificamente em Pernambuco, tornou pertinente o desenvolvimento desta pesquisa, visando, sobretudo, comparar as medidas antropométricas da envergadura e circunferência braquial, objetivando utilizá-las como indicadores que possam substituir o IMC [peso $(\mathrm{kg}) / a$ altura $\left(\mathrm{cm}^{2}\right)$ ] na avaliação do estado nutricional do idoso, contribuindo para o aprofundamento do tema em estudo e oferecendo maiores subsídios para os programas de assistência ao idoso.

\section{MÉ TO D O S}

O trabalho realizado foi do tipo transversal, em 20 instituições assistenciais de caráter filantrópico, governamental e, também, particular, das cidades do Recife, Cabo de Santo Agostinho, Vitória de Santo Antão, Olinda e Caruaru, escolhidas de forma aleatória. Este estudo foi aprovado pelo Comitê de Ética da Universidade de Pernambuco (Protocolo no 002/99), observando-se o cumprimento dos princípios éticos contidos na Declaração de Helsinki, da Word Medical Association. Em todas as instituições foi obtida autorização dos gestores, a partir de assinatura de termo de autorização e compromisso. Todos os participantes foram previamente esclarecidos pela equipe, sobre os objetivos do trabalho e as técnicas às quais seriam submetidos, e só fizeram parte da amostra os que concederam permissão para sua execução.

A amostra foi constituída por 308 idosos de ambos os sexos e com idade igual ou superior a 60 anos. Foram incluídos no estudo todos os indivíduos que se encontravam, no momento, em condições físicas e mentais, aptos a serem examinados. Na seleção da amostra não se levou em consideração a presença de doenças, fossem elas agudas ou crônicas, desde que as mesmas não interferissem na operacionalização das medidas antropométricas. Indivíduos com deformidade física ou incapacidade motora foram excluídos da análise.

A coleta de dados ocorreu no período de abril de 1998 a janeiro de 1999 com coordenação, supervisão e operacionalização dos trabalhos de campo a cargo do principal investigador. As entrevistas foram realizadas nas instituições assistenciais, sempre no período da manhã, durante os dias de semana ou nos finais de semana, de acordo com a disponibilidade do local visitado. $\mathrm{O}$ espaço físico onde ocorreu a coleta dos dados foi cuidadosamente escolhido, observando-se a regularidade das paredes e do piso para que não interferissem nos resultados. Para realização das medidas, os idosos foram despidos de suas roupas e calçados e vestidos com jalecos iguais, produzidos especificamente para este trabalho, para padronizar a interferência da roupa no peso obtido. As medidas da envergadura foram realizadas com fixação de fitas crepes na parede, que direcionavam com maior precisão a abdução dos braços dos idosos.

As medições obedeceram aos procedimentos balizados por Gibson ${ }^{15}$, Chumlea ${ }^{16} \mathrm{e}$ Chilima \& Ismail ${ }^{17}$. O instrumento da anotação dos dados foi um formulário contendo variáveis antropométricas (peso, altura, envergadura, circunferência braquial) e medidas derivadas. Os instrumentos utilizados na coleta das medidas foram: para o peso, balança eletrônica marca Filizola, modelo E-150/3P, com capacidade para $150 \mathrm{~kg}$ e precisão de $0,1 \mathrm{~kg}$; para altura, fita Stanley-ambo 01-116, com $200 \mathrm{~cm}$ de extensão e $0,1 \mathrm{~cm}$ de precisão; e, para envergadura, fita de fibra de vidro CMS Weighing/Equipament Ltda, com extensão de $200 \mathrm{~cm}$ e precisão de $0,01 \mathrm{~cm}$.

Para o diagnóstico do estado nutricional dos idosos, foram utilizados: a) o Índice de Massa 
Corporal [Peso $(\mathrm{kg}) /$ Altura $\left(\mathrm{m}^{2}\right)$ ]. Adotou-se a padronização de James et al. ${ }^{18}$, os quais sugerem como corte para normalidade, o intervalo de 18,5 a $24,9 \mathrm{~kg} / \mathrm{m}^{2}$ para o IMC; b) o Índice de Massa Corporal calculado pela altura estimada pela envergadura [Peso $(\mathrm{kg}) /$ Altura estimada $\left(\mathrm{m}^{2}\right)$ ]. Para obtenção desta medida, foram utilizadas as equações validadas no estudo de Chilima \& Ismail ${ }^{14}$ para homens: altura $(\mathrm{cm})=51,5+0,64$ (envergadura) e para mulheres: altura $(\mathrm{cm})=45,9+0,66$ (envergadura). Na avaliação da circunferência braquial foi utilizado o valor igual ou maior que 24,0 para classificar os indivíduos como adequados $^{17}$

As medições foram realizadas por equipe capacitada, utilizando-se a metodologia validada por Habicht ${ }^{19}$ e Frisancho ${ }^{20}$. Para qualificar a variação intra-observador das variáveis contínuas, foram utilizados o Erro Técnico de Medida (TEM) e o coeficiente de confiabilidade (R), indicado por Frisancho ${ }^{20}$.

Os dados foram codificados e digitados em dupla entrada e processados em microcomputador, utilizando o software Epi Info, versão $6.04 d^{21}$, com a finalidade de validar a sua consistência. Foi realizada a comparação do uso da envergadura em substituição à estatura no cálculo do IMC, com relação ao IMC calculado pela forma tradicional. Para tanto, avaliou-se a sensibilidade, definida como a proporção de indivíduos com resultados positivos (déficit ou excesso nutricional) nos índices antropométricos padrão e de comparação, e a especificidade, definida como a proporção de indivíduos com resultados negativos (adequação nutricional) para os mesmos índices antropométricos em estudo. De forma complementar, foram calculados o valor preditivo positivo e negativo dos dois métodos, com o auxílio do Programa Epitable-Epi Info, 6.04d.

\section{RES U LTA DOS}

A amostra, constituída por 308 idosos, caracterizou-se por apresentar um percentual de 67,9\% de indivíduos do sexo feminino, uma média de idade em torno de 75 anos (desvio-padrão $\mathrm{DP}=8,3$ ) e um percentual de $56,5 \%$ dos idosos, referindo que sabiam ler e escrever.

$\mathrm{Na}$ análise do estado nutricional, considerando-se a altura no cálculo do IMC, observou-se um percentual de $14,9 \%$ dos idosos classificados como desnutridos, $24,4 \%$ na faixa de sobrepeso e $9,1 \%$ de obesidade. Na utilização da envergadura em substituição à altura no cálculo do IMC, a avaliação nutricional da amostra foi de $23,1 \%$ dos idosos com desnutrição, $14,9 \%$ com sobrepeso e 5,8\% com obesidade. No diagnóstico nutricional, utilizando-se a circunferência braquial, foi encontrado um percentual de $24,4 \%$ de idosos desnutridos (Tabela 1).

A Tabela 2 apresenta a comparação dos indicadores empregados na pesquisa, na qual se verifica que o IMC (envergadura) apresentou uma sensibilidade de $93,5 \%$ para identificar os desnutridos entre os de baixo peso, respaldado pelo valor preditivo negativo de 97,7\%; e uma especificidade de 81,9\% para diagnosticar os indivíduos clinicamente adequados com valor preditivo positivo de $60,6 \%$. Na comparação do uso da envergadura, na classificação de idosos em rela-

Tabela 1. Estado nutricional de idosos institucionalizados, segundo os indicadores IMC* (altura) e IMC (envergadura) e circunferência braquial. Pernambuco, Brasil, 1999.

\begin{tabular}{lrcr}
\hline Índices & Pontos de corte & $\mathrm{n}=308$ & $\%$ \\
\hline IMC (altura) & $<18,5$ & 46 & 14,9 \\
Desnutrido & $18,5-24,9$ & 159 & 51,6 \\
Adequado & $25,0-29,9$ & 75 & 24,4 \\
Sobrepeso & 230,0 & 28 & 9,1 \\
Obesidade & $<18,5$ & 71 & 23,1 \\
IMC (envergadura) & $18,5-24,9$ & 173 & 56,2 \\
Desnutrido & $25,0-29,9$ & 46 & 14,9 \\
Adequado & $\geq 30,0$ & 18 & 5,8 \\
Sobrepeso & & & \\
Obesidade & & & \\
Circunferência braquial (cm) & $<24,0$ & 75 & 24,4 \\
Desnutrido & $\geq 24,0$ & 233 & 75,6 \\
Adequado & & & \\
\hline
\end{tabular}

* IMC: índice de Massa Corporal 
Tabela 2. Sensibilidade, especificidade e valores preditivos positivo (VPP) e negativo (VPN) do índice de massa corporal (envergadura) e da circunferência braquial em comparação ao índice de massa corporal (altura) de idosos institucionalizados. Pernambuco, Brasil, 1999.

\begin{tabular}{lcccccccc}
\hline Índices & Sensibilidade & (IC 95\%) & Especificidade (IC 95\%) & VPP & (IC 95\%) & VPN (IC 95\%) \\
\hline IMC (envergadura) & & & & & & & & \\
Desnutrido vs Adequado & 93,5 & $(81,1-98,3)$ & 81,9 & $(74,8-87,5)$ & 60,6 & $(48,2-71,7)$ & 97,7 & $(92,9-99,4)$ \\
Sobrepeso vs Adequado & 44,4 & $(32,9-56,6)$ & 96,9 & $(91,9-99,0)$ & 88,9 & $(73,0-96,4)$ & 76,0 & $(68,7-82,2)$ \\
Obesidade vs Adequado & 83,3 & $(57,7-95,6)$ & 100,0 & $(96,3-100,0)$ & 100,0 & $(74,7-100,0)$ & 97,7 & $(92,9-99,4)$ \\
Obesidade vs Sobrepeso & 60,0 & $(38,9-78,2)$ & 91,4 & $(75,8-97,8)$ & 83,3 & $(57,7-95,6)$ & 76,2 & $(60,2-87,4)$ \\
Circunferência braquial & 89,1 & $(75,6-95,9)$ & 87,0 & $(82,2-90,7)$ & 54,7 & $(42,8-66,1)$ & 97,9 & $(94,8-99,2)$ \\
\hline
\end{tabular}

IC: intervalo de confiança.

ção à condição de sobrepeso versus (vs) adequado, evidenciou-se uma sensibilidade de $44,4 \%$, com valor preditivo negativo de $76 \%$ e especificidade de $96,9 \%$, para o diagnóstico de indivíduos adequados, com valor preditivo positivo de $88,9 \%$. Na identificação de idosos classificados entre obesos vs adequados, observou-se, na análise da validação, uma sensibilidade do método de $83,3 \%$ para o diagnóstico de obesidade, com valor preditivo negativo de $97,7 \%$, e uma especificidade e valor preditivo positivo de $100,0 \%$ no diagnóstico dos idosos com condição adequada.

Com relação à classificação dos indivíduos em relação à obesidade vs sobrepeso, observou-se que o método da envergadura apresentou uma sensibilidade de $60,0 \%$ no diagnóstico de indivíduos obesos, com valor preditivo negativo de $76,2 \%$, e especificidade de $91,4 \%$ para identificação de indivíduos com sobrepeso, com valor preditivo positivo de $83,3 \%$.

Na classificação de desnutrição utilizando a circunferência braquial, verificou-se uma sensibilidade de $89,1 \%$, para o método com valor preditivo negativo de $97,9 \%$.

\section{I S C U S S Ã O}

Com relação à prevalência de desnutrição, se observou, neste estudo, um percentual de $14,9 \%$, com a utilização do IMC (altura); no trabalho de Chilimia \& Ismail ${ }^{17}$, para avaliação do estado nutricional de idosos indianos, foi evidenciado um percentual de $29,8 \%$ de desnutridos.
Mijares et al.22, estudando idosos institucionalizados em Valência, Espanha, encontraram um percentual de desnutrição de $26,8 \%$. Os diferenciais encontrados pelos autores com relação aos percentuais de desnutrição podem ser justificados pelas diferenças culturais, inerentes a cada país, em especial a Índia, pelas precárias condições de vida e de pobreza do povo indiano. Os altos percentuais de desnutrição no trabalho realizado na Espanha foram justificados pela maior dificuldade de acesso a comida, pela rotina alimentar, pela falta de preocupação em oferecer uma dieta balanceada, fatores esses característicos da vida asilar, agravados pelos problemas nutricionais inerentes ao próprio processo de envelhecimento.

Em contraponto, no estudo realizado por Perissinotto et al. ${ }^{23}$, com idosos italianos que viviam em comunidade, foi observado apenas um pequeno percentual de desnutrição (5\%), tendo sido tal resultado alusivo ao costume alimentar desse povo, o qual, culturalmente, se caracteriza por uma dieta rica em calorias, elevando, assim, os percentuais de obesidade dentro da população. Resultados semelhantes foram encontrados no trabalho de Cabrera et al. ${ }^{24}$ durante seguimento de 5 anos de idosas, encontrando um percentual de desnutrição de 4,7\%.

$\mathrm{Na}$ avaliação de sobrepeso, o presente estudo encontrou percentuais de $24,4 \%$ entre os idosos, valores baixos, se comparados com os do trabalho de Santos \& Sichieri ${ }^{25}$ no qual 50,0\% dos idosos estudados apresentaram sobrepeso; 
entretanto, as autoras ponderaram sobre os valores elevados do IMC nessa faixa etária, tendo em vista a redução da estatura e da massa magra.

Na utilização do cálculo do IMC (envergadura) para avaliação do estado nutricional, foram diagnosticados como desnutridos $23,1 \%$ do total dos 308 idosos. Chilimia \& Ismail ${ }^{17}$, utilizando a mesma metodologia aplicada no presente estudo, encontraram $48,0 \%$ de desnutridos entre os 296 idosos avaliados em Malawi. A proporção elevada de desnutrição pelo cálculo do IMC (envergadura), vista no trabalho de Chilimia \& Ismail ${ }^{17}$, pode ser justificada pelas condições de pobreza e, principalmente, do estado contínuo de miséria e fome que acompanha essa população desde a infância, persistindo na adolescência e, conseqüentemente, refletindo-se na velhice, associada a repetidas infecções, pela baixa imunidade e problemas nutricionais nas idades mais avançadas. No entanto, é plausível que a prevalência de desnutrição do presente estudo seja menor do que a encontrada por Chilimia \& Ismail ${ }^{17}$, ao considerar, sobretudo, as diferenças sócio-econômica-culturais e demográficas entre as duas populações, principalmente, pela falta de ações políticas que visem a melhorar as condições de vida da população de Malawi, incluindo a redução da fome naquele país.

Em idosos o emprego do IMC apresenta dificuldades, em função da redução da altura com a acentuação da cifose dorsal, devido à redução da massa corporal magra, ao acúmulo da gordura visceral ou subcutânea e à redução da quantidade de água no organismo. Portanto, como indicador de risco no idoso, o IMC tem sido considerado pobre, em razão de não refletir, principalmente, a distribuição regional da gordura ${ }^{26}$. No Brasil, não há estudos de base populacional em idosos, que permitam avaliar a adequação do IMC como marcador de adiposidade, entretanto facilidade de obtenção de dados de peso e estatura, bem como a sua boa correlação com a mortalidade e a morbidade justificam a utilização do IMC em estudos epidemiológicos e na prática clínica. Por outro lado, é comum idosos estarem em cadeiras de rodas ou acamados, dificultando a realização da antropometria, podendo, inclusive, levar a medidas errôneas. Nesse contexto é que se indica o uso de medidas recumbentes ${ }^{27}$, com fórmulas que podem predizer a altura, como é a medida da envergadura utilizada neste estudo.

Com relação à utilização da envergadura, os elevados percentuais de sensibilidade e valor preditivo negativo para o diagnóstico de baixo peso denotam a confiabilidade do método para o diagnóstico da desnutrição. Também, para o diagnóstico de obesidade, os percentuais de especificidade e de valor preditivo positivo confirmam a adequada utilização da envergadura no cálculo do IMC. Entretanto, para confrontar as condições de sobrepeso vs adequado e obeso vs sobrepeso, os baixos percentuais de sensibilidade e do valor preditivo negativo configuram a necessidade de métodos complementares para a confirmação do referido diagnóstico.

Na utilização da circunferência braquial para classificação do estado nutricional de idosos, observa-se um percentual total de $24,4 \%$ da amostra com desnutrição. Pieterse et al. ${ }^{28}$, em seu trabalho de avaliação nutricional de 828 idosos na Tanzânia, utilizando a circunferência braquial, encontraram um percentual de $13,6 \%$ de desnutrição, sendo atribuído este valor, ao fato de que, o HelpAge International, uma organização não governamental, permaneceu, por mais de ano, no campo de refugiados onde o estudo foi realizado, advogando em favor dos interesses dos idosos e, também, oferecendo orientação e suporte nutricional, o que levou à notória melhoria no estado nutricional dessa população, em comparação com o restante do país. Menezes \& Marucci29, em seu estudo com 305 idosos em instituições de longa permanência, concluíram que, apesar de não ser o melhor indicador de massa muscular, o perímetro do braço sofre alterações com o declínio da quantidade de massa magra, tendo em vista que ele representa o somatório das áreas constituídas pelos tecidos ósseo, muscular, gorduroso e epitelial do braço, mostrando-se reduzido com a idade, o que é mais pronunciado nas mulheres. 
Santos \& Sichieri ${ }^{30}$, em inquérito realizado com idosos no Rio de Janeiro, comparando o IMC com outros indicadores de adiposidade e localização de gordura, observaram, também, uma progressiva redução da área muscular do braço com a idade, entretanto, a medida do perímetro braquial apresentou uma alta correlação com o $I M C$, tendo sido sugerido como indicador substituto do IMC ou como medida adicional para avaliação do estado nutricional de populações.

No presente trabalho a utilização da circunferência braquial como parâmetro de avaliação nutricional de desnutrição em idosos, demonstrou uma sensibilidade de $89,1 \%$, com um valor preditivo negativo de $97,9 \%$, representando uma confiabilidade do método para o diagnóstico da desnutrição, semelhante aos valores encontrados para a envergadura em substituição à altura, no cálculo do IMC.

\section{CONCLUSÃO}

Após análise dos resultados, conclui-se que é mais elevada a prevalência de desnutrição quando se utiliza a medida da envergadura em substituição à de altura no cálculo do IMC, bem como a de circunferência braquial. O IMC (envergadura) mostrou ser um bom indicador no diagnóstico de desnutrição e de obesidade, no entanto, não se observou o mesmo em relação ao diagnóstico de sobrepeso. A medida da circunferência braquial também constitui um bom indicador para determinar desnutrição em idosos.

Embora a literatura seja clara na valorização e importância da utilização do IMC como indicador do estado nutricional, a unanimidade, contudo, entre os autores, é da necessidade contínua da busca, a partir de novas pesquisas, de métodos para estimativa de uma avaliação nutricional com maior acurácia e precisão, analisando adequação dos pontos de corte, especificamente, voltada para a população idosa.

\section{COLABORADORES}

A.M. GARCIA e P.I.C. LIRA participaram na elaboração do projeto, execução do trabalho, análise dos dados e redação do artigo. S.A.M. ROMANI participou na elaboração do projeto, análise dos dados e redação do artigo.

\section{R E F E R Ê N CIAS}

1. Kalache A, Veras R, Ramos L. O envelhecimento da população mundial: um desafio novo. Rev Saúde Pública. 1987; 21(3):200-10.

2. Ramos LR. A explosão demográfica da terceira idade no Brasil: uma questão de saúde pública. Gerontologia. 1993; 1(1):3-8.

3. Beltrão $M L$, Laurenti R. Saúde, bem-estar e envelhecimento. Rev Bras Epidemiol. 2005; 8(2): 127-41

4. Jee SH, Sull JW, Park J, Lee S-Y, Ohrr H, Guallar E, et al. Body-Mass Index and Mortality in Korean Men and Women. N Engl J Med. 2006; 355(B):779-87

5. McGee DL. Body mass index and mortality: a meta analysis based on person level data fromtwenty-six observational studies. Ann Epidemiol. 2005; 15:87-97

6. Najas MS. Padrão alimentar de idosos de diferentes estratos sócio econômicos residentes em localidade urbana da região Sudeste Brasil. Rev Saúde Pública. 1994; 28(3):187-91.

7. Chapman KM, Ham JO, Pearlman RA. Longitudinal assessment of the nutritional status of elderly veterans. J Geront. 1996; 51:261-5.

8. Otero U, Rozenfeld S, Gadelha A, Carvalho M. Mortalidade por desnutrição em idosos, região sudeste do Brasil,1980-1997. Rev Saúde Pública. 2002; 36(2):141-8.

9. Pirlich $M$, Lochs $H$. Nutrition in the elderly. Best Pract Res Clin Gastroenterol. 2001; 15(6):869-84.

10. Lukaski H. Methods for the assessment of human body composition: traditional and new. Am J Clin Nutr. 1997; 46:537-56.

11. De Luis D, Aller R, Cabezas G, Terroba C, Cuellar L. A comparison of 2 reference tables in nutritional antropometric assesment. Nutr Hosp. 2000; 15 (3):114-7.

12. Butters M, Straub M, Kraft K, Bittner R. Studies on nutritional status in general surgery patients by clinical, anthropometric, and laboratory parameters. Nutrition. 1996; 12(6):405-10.

13. Carvalho Filho ET, Papaléo Neto M. Geriatria: fundamentos, clínica, terapêutica. São Paulo: Atheneu; 2005.

14. Chapman N, Bannerman E, Cowen S, MacLennan $W$. The relationship of anthropometry and bio- 
-electrical impedance to dual energy X-ray absorptiometry in elderly men and women. Age and Ageing. 1998. 27(3):363-7.

15. Gibson RS. Principles of nutritional assessment. New York: Oxford University Press; 1990.

16. Chumlea WC, Guo S, Vellas B, Guigoz Y. Techniques of assessing muscle mass and function for epidemiological studies of the elderly. J Gerontol. 1990; 50-A:45-52.

17. Chilima DM, Ismail SJ. Anthropometric characteristics of older people in rural Malawi. Eur J Clin Nutr. 1998; 52:643-9.

18. James WPT, Ferro-Luzzi A, Waterlow C. Definition of chronic energy deficiency in adults. Eur J Clin Nutr. 1998; 42:969-81.

19. Habicht JP. Estandarizacion de métodos epidemiológicos cuantitativos sobre el terreno. Bol Of Sanit Panam. 1974; 76(5):375-81.

20. Frisancho AR. Anthropometric standards for the assessment of growth and nutritional status. Michigan: University Michigan Press; 1990.

21. Dean AG, Dean JA, Coulombier D, Brendel KA, Smith DC, Burton $\mathrm{AH}$, et al. Epi Info [computer program]. Version 6: a word processing, database, and statistics program of epidemiology on micro-computers. Atlanta, Gerogia: Centers os Disease Control and Prevention; 1994.

22. Mijares A, Taberner R, Triguero M, Fandos J, Garcia A, Suarez-Varela M. Prevalencia de malnutrición entre ancianos institucionalizados en la Comunidad Valenciana. Med Clin. 2001; 117(8): 289-94.
23. Perissinotto E, Pisent C, Sergi G, Grigoletto F. Anthropometric measurements in the elderly: age and gender differences. Br J Nutr. 2002; 87(2): 177-86.

24. Cabrera M, Wajngarten M, Gebara O, Diament J. Relação do índice de massa corporal, da relação cintura-quadril e da circunferência braquial com a mortalidade em mulheres idosas: seguimento de 5 anos. Cad Saúde Pública. 2005; 21(3): 767-77.

25. Santos D, Sichieri R. Índice de massa corporal e indicadores antropométricos de adiposidade em idosos. Rev Saúde Pública. 2005; 39(2):163-8.

26. Sampaio LR, Figueiredo VC. Correlação entre o índice de massa corporal e os indicadores antropométricos de distribuição de gordura corporal em adultos e idosos. Rev Nutr. 2005; 18(1):53-61.

27. Cervi A, Franceschini SC, Priori SE. Análise crítica do uso de índice de massa corporal para idosos. Rev Nutr. 2005; 18(6):765-75

28. Pieterse $S$, Manandhar $M$, Ismail $S$. The nutritional status of older Rwandan refugees. Pub Health Nutr. 1998; 1(4):259-64.

29. Menezes T, Marucci M. Antropometria de idosos residentes em instituições geriátricas Fortaleza, CE. Rev Saúde Pública. 2005; 39(2):169-75.

Recebido em: 11/4/2006

Versão final reapresentada em: 30/10/2006 Aprovado em: 22/12/2006 